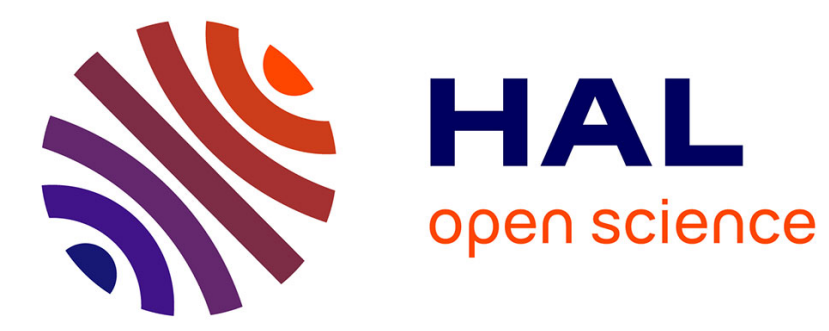

\title{
Détermination expérimentale de quelques caractéristiques optiques d'un système double prisme magnétique-miroir électrostatique
}

M. Leleyter, G. Slodzian, R. Quettier

\section{- To cite this version:}

M. Leleyter, G. Slodzian, R. Quettier. Détermination expérimentale de quelques caractéristiques optiques d'un système double prisme magnétique-miroir électrostatique. Revue de Physique Appliquée, 1972, 7 (2), pp.117-125. 10.1051/rphysap:0197200702011700 . jpa-00243602

HAL Id: jpa-00243602

https://hal.science/jpa-00243602

Submitted on 1 Jan 1972

HAL is a multi-disciplinary open access archive for the deposit and dissemination of scientific research documents, whether they are published or not. The documents may come from teaching and research institutions in France or abroad, or from public or private research centers.
L'archive ouverte pluridisciplinaire HAL, est destinée au dépôt et à la diffusion de documents scientifiques de niveau recherche, publiés ou non, émanant des établissements d'enseignement et de recherche français ou étrangers, des laboratoires publics ou privés. 
Classification

Physics abstracts

06.20

\title{
DÉTERMINATION EXPÉRIMENTALE DE QUELQUES CARACTÉRISTIQUES OPTIQUES D'UN SYSTËME DOUBLE PRISME MAGNÉTIQUE- MIROIR ÉLECTROSTATIQUE
}

\author{
M. LELEYTER $\left(\mathrm{M}^{\text {elle }}\right)$ \\ Faculté des Sciences, 33, rue Saint-Leu, 80-Amiens \\ G. SLODZIAN et R. QUETTIER
}

Laboratoire de Physique des Solides (*), Bâtiment 510, Université de Paris-Sud, 91-Orsay

(Reçu le 29 février 1972)

\begin{abstract}
Résumé. - Nous avons mis au point une méthode pratique pour étudier expérimentalement les propriétés optiques du premier ordre (focalisation) dans la première traversée de l'aimant du microanalyseur ionique (constitué par un double prisme magnétique à champ uniforme associé à un miroir électrostatique).

On détermine ainsi le point stigmatique réel du prisme à quelques dixièmes de millimètre près. Le dispositif utilisé est assez précis pour qu'on puisse mesurer les aberrations d'ouverture du second ordre au cross-over intermédiaire.

Par ailleurs, en étudiant expérimentalement le miroir avec l'analyseur complet, nous localisons le centre du miroir convexe équivalent à quelques dixièmes de millimètre près également, ainsi que son sommet, et nous mettons en évidence l'influence de l'énergie des particules sur la position du centre du miroir.

Ceci permet d'obtenir une meilleure résolution en masse et d'améliorer la qualité des images de distribution données par l'analyseur.

Abstract. - We have devised a method to study experimentally the first-order optical features (focusing) of the first part of the deflecting magnet used in an ion-microanalyser (which contains a double homogeneous magnetic prism associated with an electrostatic mirror). The real stigmatic point of the prism is determined within a few tenths of a millimeter with this method, which is precise enough to let us measure the second-order aperture aberrations at the intermediate crossover. By studying experimentally the mirror with the whole analyser, we have localized the equivalent convex mirror centre within a few tenths of a millimeter, as well as its apex, and we have also seen the influence of the particle energy on the position of the mirror centre. These results permit us to find the best position for the mass selection slit and thus obtain the best mass resolution, as well as improve the resolving limit on the ionic microanalyser images.
\end{abstract}

I. Introduction. - Dans un premier article [1], nous avions calculé les aberrations du second ordre du microanalyseur ionique [2] ainsi que celles d'autres systèmes apparentés. Nous avions utilisé une méthode géométrique faisant intervenir deux simplifications fondamentales : tout d'abord, nous n'avions pas tenu compte du champ de fuite de l'aimant du microanalyseur; en second lieu nous supposions le miroir électrostatique exempt d'aberration.

Toutefois, nous avions indiqué par la suite [3] que les aberrations chromatiques du miroir pouvaient induire des effets très importants, en particulier ceux

(*) Associé au CNRS. de la variation du rayon du miroir équivalent avec l'énergie des particules.

Par ailleurs, le calcul donne pour les aberrations d'ouverture du second ordre au cross-over de la première traversée du prisme, un signe opposé au signe déterminé expérimentalement. Matsuda [4] a fait des calculs théoriques d'aberrations du deuxième ordre dans le cas de spectrographes de masse, et il a trouvé que certaines d'entre elles pouvaient effectivement changer de signe selon que le calcul tenait ou non compte du champ de fuite de l'aimant. Enge [5] indique que les aberrations d'ouverture varient avec la courbure des faces polaires d'entrée et de sortie du prisme magnétique, alors que Woll- 
nik [6], lui, trouve que seule, l'une d'elles varie avec le champ de fuite.

La connaissance de la position du cross-over et des aberrations qui l'entachent est nécessaire lorsqu'on veut donner à la fente de sélection sa largeur optimum. De plus, nous devons placer le centre du miroir au même endroit que la fente si nous voulons que le champ imagé ne soit pas coupé lorsqu'on cherche précisément à donner cette largeur optimum à la fente. Enfin, la réduction de ces aberrations par l'utilisation de cales courbes, placées sur les pôles de l'aimant, permettrait d'améliorer le filtrage en masse.

Nous avons donc cherché à déterminer avec précision la position réelle du cross-over donnée par la première traversée, la position du centre du miroir et une forme de cale qui permettraient de réduire les aberrations. Par ailleurs, comme le faisceau secondaire est formé d'ions présentant une certaine dispersion d'énergie, nous nous sommes attachés à l'étude des défauts chromatiques essentiellement au niveau du miroir.

Nous exposons pour commencer, la méthode utilisée dans cette étude expérimentale des propriétés optiques de l'appareil ; la deuxième partie concerne l'examen des caractéristiques optiques essentielles de la première traversée du prisme magnétique. Enfin, nous étudions le système global, et en particulier, l'influence des propriétés du miroir sur le faisceau émergent.

II. Principe de la recherche des éléments optiques des premier et deuxième ordres. - 1) MÉTHODE. Pour déterminer les propriétés optiques d'un système, il faut suivre la marche de trajectoires ou de rayons lumineux particuliers : après avoir traversé le système optique, les trajectoires issues d'un point source se rencontrent autour d'un «point image». Pour localiser ce point et déterminer les "écarts » des trajectoires autour de cette position, nous utilisons une méthode qui consiste à couper le faisceau par une lame et à observer l'ombre qui en résulte sur un écran placé plus loin. Il est facile de voir que selon la position relative de la lame et du point image, l'ombre apparaît d'un côté ou de l'autre. On a là, un moyen simple et bien connu de trouver avec précision la position du point image. Lorsqu'on fait intervenir les aberrations, la forme de l'ombre est plus ou moins compliquée, mais on peut simplifier le problème en isolant, dans le faisceau issu du point source, des pinceaux de trajectoires au moyen d'un diaphragme.

La difficulté qui reste à surmonter dans notre cas est de trouver un écran convenable pour observer les ions. L'utilisation d'un convertisseur d'image [7] consiste à remplacer en quelque sorte l'écran par une plaque métallique (cathode) que les ions viennent frapper ; les électrons issus de ces impacts sont ensuite focalisés en une image de la surface de la cathode. Le schéma du fonctionnement d'un convertisseur d'images se trouve sur la figure 1, mais ce qui est représenté sur cette figure, c'est le convertisseur qui transforme les images d'ions secondaires en images électroniques. En définitive, on observe sur un écran fluorescent, une image électronique qui donne la distribution de la trace, sur la cathode, des pinceaux de trajectoires.

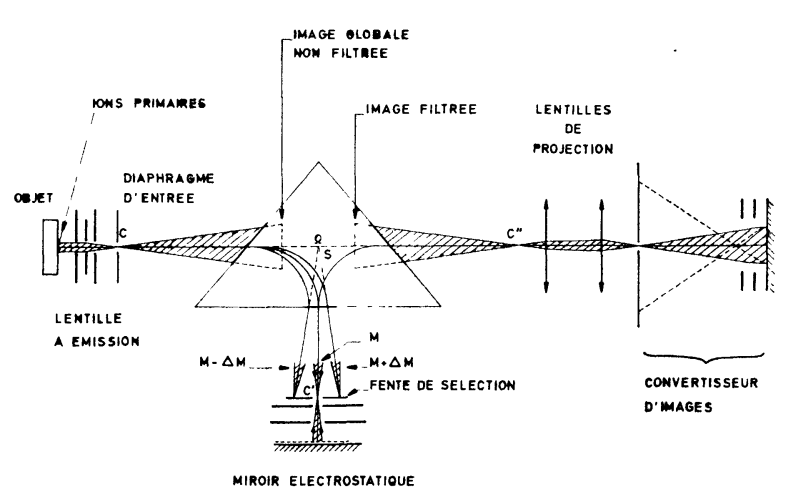

Fig. 1. - Schéma du microanalyseur. $\Omega$ : focale achromatique ; $\mathrm{S}$ : sommet du miroir. Pour l'étude des aberrations en $\mathrm{C}^{\prime}$, l'objet est remplacé par un filament et la fente de sélection par un dispositif de coupure.

2) Montage expérimental. - Sur la figure 1 est schématisée la constitution de l'appareil que nous avons déjà décrit par ailleurs.

a) Particules utilisées. - Pour simplifier l'étude expérimentale, nous avons utilisé un faisceau composé de particules identiques et monocinétiques; il s'agit ici d'ions alcalins émis par le filament de tungstène d'un canon à électrons qui porte une boule d'aluminosilicate du métal correspondant ( $\mathrm{Li}, \mathrm{Na}, \mathrm{K}$ ou $\mathrm{Cs}$ ) que l'on chauffe. A l'aide d'une lentille à émission qui joue le rôle de condenseur, on obtient un faisceau d'ions primaires $\mathrm{M}^{+}$de même masse et de même énergie, nécessaire pour étudier expérimentalement la focalisation et les aberrations géométriques en s'affranchissant du chromatisme.

La lentille à émission focalise les ions $\mathbf{M}^{+}$en un cross-over au niveau duquel on place un diaphragme de contraste de $200 \mu, 100 \mu$ ou $50 \mu$ (diaphragme d'entrée sur la figure 1).

b) Isolement des pinceaux de trajectoires à l'entrée. Sur le trajet des ions issus de ce cross-over, on interpose un diaphragme $\mathrm{D}$ percé de trous de 100 à 110 microns de diamètre disposés comme l'indique la figure 2. L'une des lignes (trous no 1 à 7) est située dans le plan de symétrie de l'appareil (première section principale), l'autre ligne (trous $n^{0} 1^{\prime}$ à $7^{\prime}$ ) est parallèle au vecteur induction $\mathbf{B}$ (deuxième section principale).

On prélève ainsi dans le faisceau incident des pinceaux qui matérialisent approximativement des trajectoires de particules faisant avec l'axe des angles $\alpha_{0}, 2 \alpha_{0}, 3 \alpha_{0}$ (l'angle d'ouverture maximum est $2 \alpha \sim 2 \times 10^{-2}$ radian). 


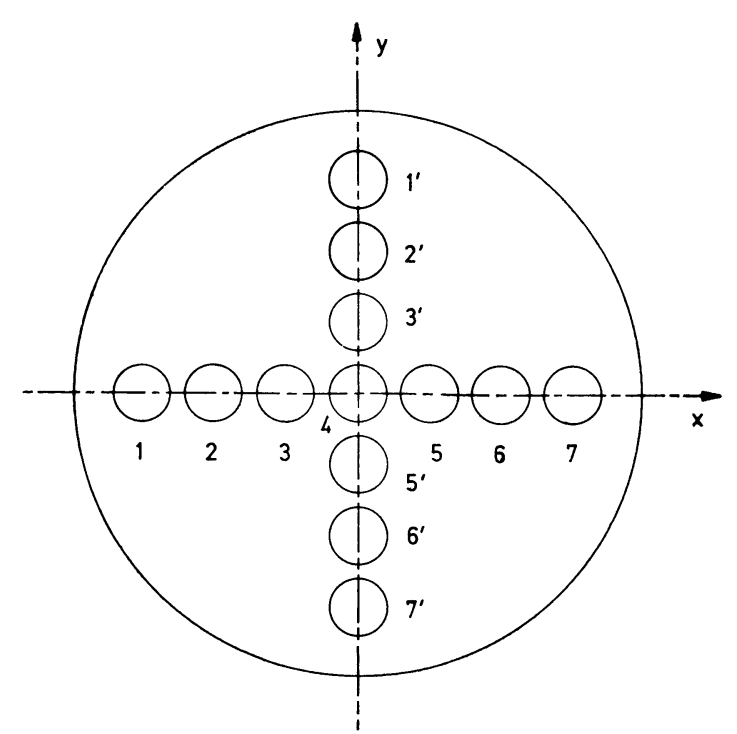

Fig. 2. - Diaphragme D : les 13 trous qui découpent le faisceau incident ont un diamètre de 100 à $110 \mu$ et sont disposés sur des diamètres de : $0,7 \mathrm{~mm}$ : trous $3,5,3^{\prime}$ et $5^{\prime} ; 1,4 \mathrm{~mm}$ : trous $2,6,2^{\prime}$ et $6^{\prime} ; 2,1 \mathrm{~mm}:$ trous $1,7,1^{\prime}$ et $7^{\prime}$.

La localisation, à la sortie de l'appareil, de ces divers rayons, permet de situer facilement les focales du prisme, de rechercher le stigmatisme et d'étudier les aberrations d'ouverture.

c) Dispositif de coupure. - Sortant de l'aimant, le faisceau d'ions rencontre un dispositif de coupure qui est constitué principalement par une lame de platine L d'épaisseur $0,04 \mathrm{~mm}$, percée d'un trou rectangulaire $\mathrm{T}$ (de $2 \mathrm{~mm} \times 1 \mathrm{~mm}$ ), dont le plan est perpendiculaire à l'axe optique (Fig. 3). Cette lame est portée par un support qu'on peut déplacer sous vide dans les trois directions de l'espace :

- parallèlement à l'axe optique, entre l'aimant et le miroir électrostatique (mouvement 1 sur la figure 3) ;

— perpendiculairement à cet axe (c'est-à-dire dans le plan xy de la lame $\mathrm{L}$ ) :

- soit dans le plan radial (coupure du faisceau par les grands côtés de T parallèles à $\mathrm{B}$ : mouvement 2);

- soit dans la direction de l'induction $\mathbf{B}$ dans l'entrefer (coupure par les petits côtés de $\mathrm{T}:$ mouvement $\underline{3}$ ).

Un diaphragme $(400 \mu)$ peut être placé à la sortie de l'aimant, après la première traversée, pour aligner correctement le faisceau émergent, c'est-à-dire pour que les bords du trou $\mathrm{T}$ se trouvent toujours à la même cote par rapport à l'axe optique lorsqu'on fait subir

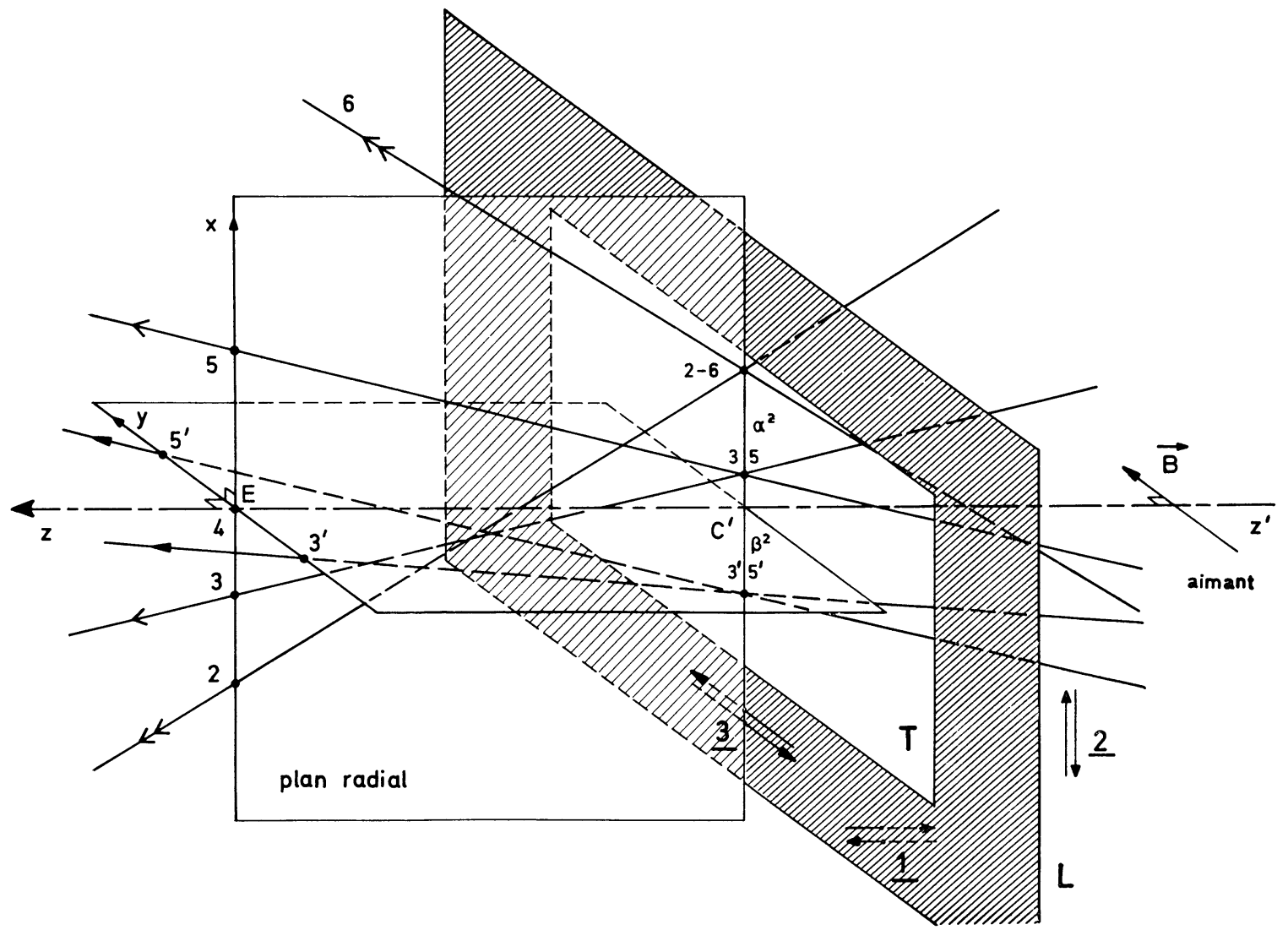

FIG. 3. - Dispositif de coupure pour l'analyse du faisceau émergent. La lame L (percée du trou rectangulaire T) est perpendiculaire à l'axe optique $\mathrm{z}^{\prime} \mathrm{z}$ du prisme magnétique (rayon 4). $\mathrm{C}^{\prime}:$ centre du cross-over de sortie; $\mathrm{xEy}:$ plan de l'écran sur lequel on observe ici les taches $2,3,4$ et 5 (rayons du plan radial) et $3^{\prime}, 5^{\prime}$ (rayons hors du plan radial). Les autres rayons ne sont pas représentés.

- Aberrations : en $\alpha^{2}=$ distance du « point 3-5» ou du «point 2-6» à $\mathrm{C}$; en $\beta^{2}=$ distance du «point $3^{\prime}-5^{\prime}$ » à $\mathrm{C}^{\prime}$.

- Mouvements de la lame $L$ : 1 : parallèlement à l'axe $\mathrm{z}^{\prime} \mathrm{z}$, pour chercher le point $\mathrm{C}^{\prime} ; 2$ : dans le plan de $\mathrm{L}$, parallèlement à l'axe x ; il détermine les aberrations en $\alpha^{2}$ et $\beta^{2}$ (sur la figure $A \alpha^{2}>0$ et $\left.B \beta^{2}<0\right)$. 3 : dans le plan de L, parallèlement à $\mathbf{B}$; il détermine les aberrations en $C \alpha \beta$ portées sur l'axe y passant par $\mathrm{C}^{\prime}$ (non représentées ici). 
à la lame $\mathrm{L}$ une simple translation parallèlement à cet axe (mouvement 1 ).

Les études ont d'abord été faites directement après la première traversée, en supprimant le miroir et en recevant les pinceaux d'ions sur un convertisseur d'images analogue à celui représenté sur la figure 1 . Ensuite, on les a poursuivies en utilisant le microanalyseur complet, ce qui donnait de meilleures conditions d'observation, mais compliquait un peu l'analyse des phénomènes.

III. Première traversée : recherche des focales et étude du stigmatisme. - En général, les trajectoires du plan radial sont focalisées en un point différent du point de focalisation des rayons de la deuxième section principale car le prisme est astigmatique, et on observe deux focales qu'on peut localiser à l'aide $\mathrm{du}$ dispositif de coupure décrit plus haut.

1) Structure du faisceau dans Le Plan Radial. Lorsqu'on coupe le faisceau en déplaçant la lame $\mathrm{L}$ dans son plan perpendiculairement à B (mouvement 2), on observe la disparition des taches correspondant aux pinceaux isolés par les trous du diaphragme D situés dans le plan radial.

Pour une position quelconque de $\mathrm{L}$ le long de l'axe optique $\mathrm{z}$, les sept taches s'éteignent les unes après les autres. On note leur ordre de disparition ainsi que la distance séparant approximativement les positions de $\mathrm{L}$ donnant les extinctions successives, pour diverses abscisses de la lame $\mathrm{L}$ le long de l'axe $\mathrm{z}$.

L'examen du tracé des rayons $2,3,4,5$ et 6 sur la figure 3 nous montre qu'on ne peut pas obtenir l'extinction des sept taches (les deux rayons extrêmes n'ont pas été représentés) en même temps. Le système est monté de telle manière que son axe optique coïncide avec la trajectoire du pinceau isolé par le trou 4 ; la position de la focale radiale est définie par l'intersection des rayons correspondant aux taches symétriques 1 et 7 , ou 2 et 6 ou encore 3 et 5 . Lorsqu'on réalise ces extinctions, le diaphragme d'analyse $\mathrm{L}$ est alors dans le plan de la focale radiale (c'est le cas représenté figure 3), mais du fait de l'existence des aberrations, les points d'intersection de ces rayons ne sont pas confondus et se situent même en dehors de l'axe optique (aberrations en $\alpha^{2} ; \alpha$ : demi-angle d'ouverture du faisceau dans le plan radial) : " points » 2-6 et 3-5 sur la figure 3 .

2) Focalisation AXIALE. - On procède exactement de la même manière pour trouver la focale axiale, mais en cherchant cette fois l'extinction simultanée des sept taches $1^{\prime}$ à $7^{\prime}$ correspondant aux trous du diaphragme $\mathrm{D}$ situés sur la ligne parallèle à $\mathbf{B}$, ce que l'on obtient en déplaçant la lame $\mathrm{L}$ dans son propre plan parallèlement à $\mathbf{B}$ (mouvement $\underline{3}$ ) : extinction par exemple des taches $3^{\prime}$ et $5^{\prime}$ par coupure des pinceaux au "point » $3^{\prime}-5^{\prime}$ sur la figure 3 . La position de $\mathrm{L}$ donnant l'extinction simultanée des taches est aussi celle de la focale axiale.
Pour obtenir le stigmatisme, c'est-à-dire la coïncidence des deux focales radiale et axiale, on retouche un peu l'angle d'incidence du faisceau à son entrée dans le prisme. Ce faisant, les focales se déplacent (surtout la focale axiale), et en retouchant de nouveau l'angle d'entrée, on arrive à obtenir le stigmatisme dans la première traversée du prisme du microanalyseur, et la position de la lame $\mathrm{L}$ matérialise alors l'emplacement des deux focales, donc du point stigmatique. La détermination des focales et du point stigmatique réel se fait ainsi à mieux qu'un millimètre (en fait, à quelques dixièmes de millimètre près). Les mesures sont bien reproductibles et assez fines pour mettre en évidence et mesurer les aberrations d'ouverture du second ordre au cross-over de sortie $\mathrm{du}$ prisme.

3) Mesure des aberrations D'OUVerture DU SECOND ORDRE. - Le cross-over intermédiaire $\mathrm{C}^{\prime}$ de l'analyseur (Fig. 1) n'est en fait que la pupille de sortie du «premier prisme». Les aberrations d'ouverture du deuxième ordre qui sont données par les coordonnées du point d'intersection d'un rayon émergent, issu du centre $\mathrm{C}$ du cross-over d'entrée et faisant avec l'axe optique les angles $\alpha$ et $\beta$ respectivement dans le plan radial et dans la deuxième section principale, avec le plan perpendiculaire à l'axe en $\mathrm{C}^{\prime}$, ont pour expressions :

$$
\begin{array}{ll}
\frac{\Delta x}{R_{0}}=A \alpha^{2}+B \beta^{2} & \text { (plan radial) } \\
\frac{\Delta y}{R_{0}}=C \alpha \beta & \left(2^{\mathrm{e}} \text { section principale }\right)
\end{array}
$$

où $R_{0}$ est le rayon de courbure de la trajectoire moyenne dans l'aimant.

Le dispositif de coupure permet de déterminer la structure du faisceau, et par suite le signe des trois coefficients d'aberrations $A, B$ et $C$.

a) Aberrations dans le plan de symétrie. - On trouve ainsi que les coefficients $A$ et $B$ sont de signes différents et que l'on a $A>0$ et $B<0$ (avec un axe x orienté comme sur la figure 3 , de telle sorte que le centre de courbure de la trajectoire dans l'aimant soit situé vers les $\mathrm{x}$ négatifs). Les valeurs mesurées. sont données dans le tableau $I$; pour un rayon de courbure $R_{0}$ d'environ $110 \mathrm{~mm}$ et une demi-ouverture du pinceau $\alpha=\beta=10^{-2}$ radian à l'entrée du prisme, on mesure $R_{0} A \alpha^{2} \sim 70 \mu$ et $R_{0} B \beta^{2} \sim 105 \mu$, d'où les valeurs des coefficients.

\section{TABLEAU I}

$\begin{array}{crrc}\begin{array}{c}\text { Coefficients } \\ \text { d'aberrations }\end{array} & A & B & C \\ \text { théoriques } & - & - & - \\ \text { référence [1] } & -3,5 & +0,6 & -17 \\ \text { mesurés sans cale } & 6,3 & -9,5 & -29 \\ \text { mesurés avec cales } & -0,9 & -1,35 & -14,5\end{array}$


b) Aberrations d'ouverture en dehors du plan de symétrie. - Nous avons aussi mesuré les aberrations du deuxième ordre en $\alpha \beta$, donc pour des rayons quelconques (en fait, nous avons pris des rayons tels que $\alpha=\beta$ en faisant tourner le diaphragme D dans son plan de $\pi / 4$ de manière à disposer, pour cette série de mesures, les deux lignes de trous suivant les bissectrices des axes $\mathrm{x}$ et $\mathrm{y}$ du plan de D) : nous avons obtenu une aberration de $160 \mu$ pour une ouverture $\alpha^{\prime}=\beta^{\prime}=\sqrt{2} / 2 \times 10^{-2}$ radian; le coefficient $C$ mesuré est négatif (tableau I), c'est-à-dire que l'effet de l'aberration pour des rayons hors du plan de symétrie est de déplacer le point de rencontre de deux rayons symétriques par rapport à l'axe vers les y négatifs (axes de la figure 3).

c) Comparaison avec les résultats théoriques. La comparaison des deux premières lignes du tableau I souligne la discordance entre les signes théoriques et expérimentaux des coefficients $A$ et $B$ (plan radial). Un tel résultat pourrait provenir du fait que la faible largeur des pièces polaires de l'aimant par rapport à l'épaisseur de l'entrefer (elles n'ont pas en effet l'allure prismatique de la figure 1 , mais sont taillées de manière à épouser la forme de la trajectoire moyenne) conduit à une structure de champ où les lignes d'égale induction sont convexes en dehors de l'aimant (concavité tournée vers l'intérieur des pièces polaires).

Nous avons donc placé à la sortie de l'entrefer des cales de fer doux de forme cylindrique (l'axe du cylindre étant contenu dans le plan de symétrie) dont la concavité est tournée vers l'extérieur du prisme, dans le but de redresser les lignes d'égale induction du champ de fuite et de les rendre parallèles à la face de sortie. Dans ces nouvelles conditions, pour un rayon de courbure des cales $R=75 \mathrm{~mm}\left({ }^{1}\right)$, on a de nouveau mesuré les aberrations d'ouverture (troisième ligne du tableau I) : le coefficient $A$ a changé de signe et les aberrations ont diminué ; pour les mêmes valeurs que précédemment, on a $R_{0} A \alpha^{2} \sim 10 \mu$, $R_{0} B \beta^{2} \sim 10$ à $15 \mu$ et $R_{0} C \alpha^{\prime} \beta^{\prime} \sim 80 \mu$.

4) Discussion. - Une étude plus poussée et plus précise des trajectoires à la sortie de la première traversée à l'aide du dispositif de coupure, a mis en évidence le fait que l'intersection de rayons du plan radial deux à deux symétriques par rapport à l'axe (rayon 4), ne se produit pas à la même abscisse le long de l'axe. La figure 4 reproduit la structure du faisceau à la sortie telle qu'on l'observe réellement avec notre appareil ; il faut en effet déplacer la lame $L$ d'environ $6 \mathrm{~mm}$ le long de l'axe pour passer de l'extinction simultanée des taches extrêmes 1 et 7 du plan radial à celle des deux taches centrales 3 et 5 , et cela qu'il y ait ou non des cales cylindriques à la sortie de l'aimant. La focale radiale n'est plus à proprement

(1) Diverses formes de cales ont été essayées ; le meilleur résultat a été obtenu avec le rayon de $75 \mathrm{~mm}$. parler définie; nous avons choisi, pour définir la focale radiale dans ce cas, l'intersection des rayons centraux 3 et $5\left(^{2}\right)$.
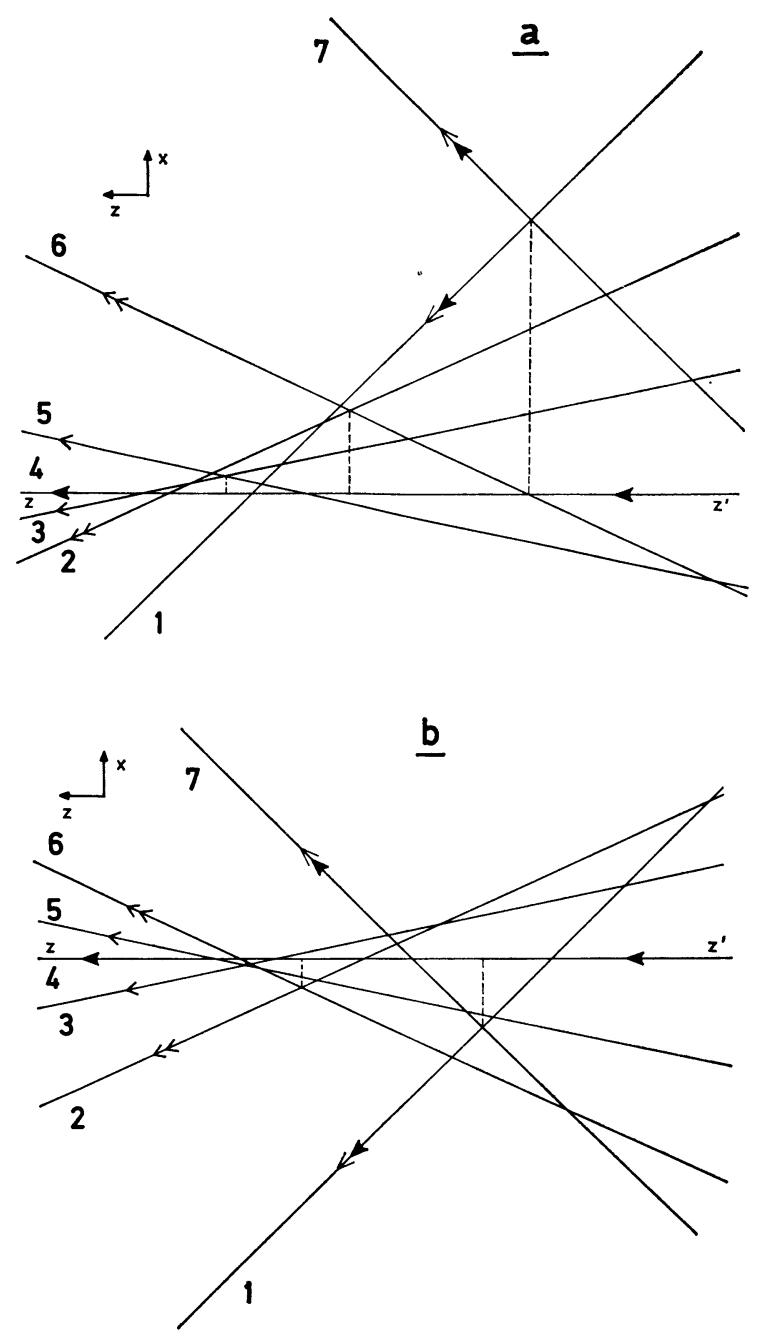

Fig. 4. - Marche des rayons à la sortie de l'aimant et aberrations en $\alpha^{2} . z^{\prime} z$ : axe optique de l'aimant, matérialisé par le rayon 4.

Fig. $a$ : aimant sans cale de fer doux à la sortie : $A \alpha^{2}>0$. Fig. $b$ : aimant avec cales de fer doux de rayon $75 \mathrm{~mm}: A \alpha^{2}<0$. (Par suite d'une certaine inhomogénéité de l'induction $B$ dans l'aimant, les rayons marginaux 1 et 7 , moyens 2 et 6 et centraux 3 et 5 ne se coupent pas sur le même axe $\mathbf{x}$ ).

Si l'on suppose que le champ magnétique à l'intérieur de l'aimant n'est pas tout à fait uniforme dès que l'on s'écarte un peu de l'axe, on peut déterminer par un calcul rapide l'abscisse du point de rencontre avec l'axe, d'une trajectoire du plan radial issue de $C$

(2) La structure du faisceau que nous avons relevée (en particulier l'inversion du signe des aberrations) pourrait s'expliquer sans qu'il soit nécessaire de faire appel à la forme du champ de fuite si l'on admet que notre aimant présente une certaine inhomogénéité de champ; les cales placées à la sortie auront simplement compensé cet effet. L'action simultanée des deux causes n'est pas exclue. 
et faisant avec l'axe un angle $\alpha$, qui traverse l'entrefer dans une zone où l'induction est $B=B_{0}+\Delta B\left(B_{0}\right.$ : induction le long de la trajectoire moyenne de rayon $R_{0}$ dans l'aimant), et dont le rayon de courbure est alors $R=R_{0}+\Delta R\left(\Delta R / R_{0}=\Delta B / B_{0}\right)$.

Soit $D$ la distance de ce point de rencontre à la face de sortie de l'aimant. On trouve :

$$
D=\frac{-\left(\Delta R / R_{0}\right)-\alpha(2 t+1)}{\left(\Delta R / R_{0}\right)-2 \alpha}
$$

où $t=\operatorname{tg} \varepsilon, \varepsilon$ angle d'entrée du faisceau dans le prisme : $t=0,843$. L'angle $\alpha$ est au maximum égal à $10^{-2}$ radian. Pour causer une variation de $D$ de $6 \mathrm{~mm}$, c'està-dire une variation relative de $6 \%$, on trouve qu'il suffirait d'une modification de rayon due à une inhomogénéité de $B$ de $5 \times 10^{-4}$ qui est très plausible dans le cas de l'appareil.

Un essai rapide avec un autre aimant du même type monté sur un autre appareil nous a montré que cet effet ne se produisait plus et que les points de rencontre « 3-5», « 2-6» et « 1-7» étaient bien dans le même plan perpendiculaire à l'axe en $C^{\prime}$ comme dans le cas de la figure 3 (ce plan est donc celui de la focale radiale définie ici sans ambiguité). De plus, le signe des coefficients $A$ et $B$ était cette fois le même que le signe prévu théoriquement.

L'étude des aberrations au cross-over intermédiaire $C^{\prime}$ est importante car c'est à ce niveau qu'on dispose les lames de sélection des masses et de la bande d'énergie. La largeur de la fente de sélection est déterminée par la largeur du cross-over, compte tenu des aberrations, ceci pour conserver un éclairement uniforme sur tout le champ imagé. La définition en masse sera donc d'autant meilleure que les aberrations au cross-over seront plus réduites.

IV. Détermination expérimentale du centre et du sommet du miroir. Système complet. - La structure du miroir électrostatique est essentiellement celle d'une lentille à émission à trois électrodes, dont la cathode est portée à une tension légèrement positive par rapport à la tension d'accélération des ions.

Le centre du miroir doit être parfaitement localisé, de manière à pouvoir le faire coïncider avec le crossover $C^{\prime}$ du prisme : dans ces conditions, la fente de sélection ne risque pas d'intercepter des rayons réfléchis et de faire ainsi apparaître des ombres sur l'image filtrée.

Le microanalyseur est réglé de telle sorte qu'il soit complètement achromatique après la deuxième traversée du prisme, ce qui se produit lorsque le sommet (virtuel) du miroir convexe équivalent coïncide avec la focale achromatique $\Omega$ du «premier prisme ». Il faut donc aussi localiser le sommet du miroir.

1) RÉGLAGE DU SOMMET S DU MIROIR SUR LA FOCALE ACHROMATIQUE $\Omega$. - On obtient cette coïncidence
(Fig. 1) par action sur l'excitation du wehnelt du miroir jusqu'à ce qu'une faible variation de l'induction $B$ ne provoque plus aucun déplacement de l'image sur l'écran de la partie image; l'image est alors achromatique. En effet, cela veut dire que, quel que soit le rayon des trajectoires dans le prisme, l'image reste inchangée.

Cette image est celle d'une grille de cuivre de 100 microns de pas sur laquelle on a déposé des fils de plexiglass très ténus et rendus conducteurs par évaporation sous vide d'une mince couche d'or. Le réglage de la position du sommet $S$ sur la focale achromatique du "premier prisme» est contrôlé par photographie de l'image pour des valeurs de l'induction : $B, B+\Delta B, B-\Delta B$ où $\Delta B$ correspond à une bande passante d'énergie des ions de \pm 5 volts. On a mesuré ainsi le déplacement d'un détail fin par rapport à un point fixe sur les photographies (Fig. 5) : il est pratiquement négligeable et correspond à un décalage, ramené à l'objet, d'environ 0,1 micron. On peut ainsi régler la position du sommet sur la focale achromatique au dixième de micron près (aberration chromatique introduite par le filtrage de l'aimant).

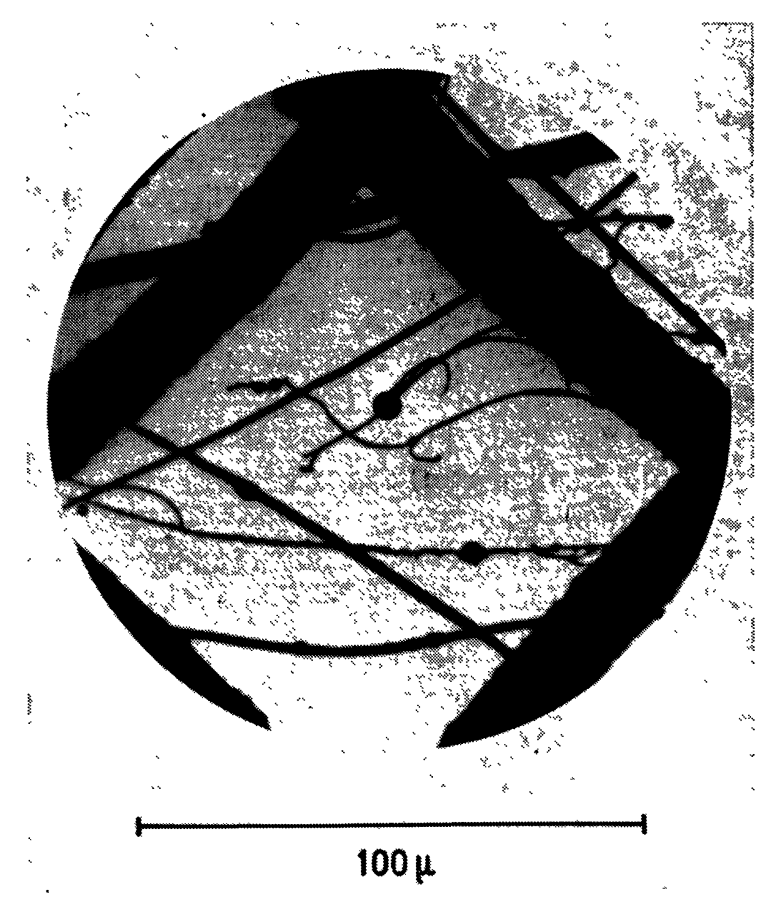

Fig. 5. - Image de fils de plexiglass donnée par l'analyseur. Les fils sont déposés sur une grille de cuivre de $100 \mu$ de pas. Le fil le plus fin a une épaisseur inférieure à un micron.

2) DÉTERMINATION DU CENTRE $\Gamma$ DU MIROIR. - a) Principe. - Le principe de la recherche du centre $\Gamma \mathrm{du}$ miroir est simple : d'après les expériences précédentes, on connait la position du point stigmatique $\mathrm{C}^{\prime}$, cross-over de sortie de la première traversée. A l'aide 
de la lame $\mathrm{L}$, on recherche l'image $\mathrm{C}_{1}^{\prime}$ de $\mathrm{C}^{\prime}$ donnée par le miroir électrostatique. Ensuite, on amène $C_{1}^{\prime}$ en coïncidence avec $C^{\prime}$ par déplacement en bloc du miroir mobile. Lorsque $\mathrm{C}_{1}^{\prime}$ et $\mathrm{C}^{\prime}$ sont confondus, ils le sont également avec le centre $\Gamma$. La position de la lame $\mathrm{L}$ donne alors celle de $\Gamma$.

Il convient de remarquer que la lame $L$ coupe non seulement des trajectoires venant directement de l'aimant, mais aussi des trajectoires réfléchies par le miroir et qui viennent frapper la lame sur leur chemin de retour. Tout se passe comme si les trajectoires ioniques étaient arrêtées, à la fois par le bord de la lame $L$ qui coupe le faisceau et par son image $L^{\prime}$ donnée par le miroir. Néanmoins, si l'on examine la figure 6 , on peut en déduire qu'en général, on ne peut pas observer directement l'image $C_{1}^{\prime}$ de $C^{\prime}$. L'exploration du faisceau, compte tenu des trajets aller et retour, conduit à la détermination des points $A$ et $B$ (Fig. $6 a$ ) qui ont une position quelconque par rapport à $\Gamma$ si le champ magnétique n'est pas centré de manière à faire coïncider l'axe optique du faisceau sortant du prisme avec un axe du miroir.

On commence donc par «centrer» l'induction
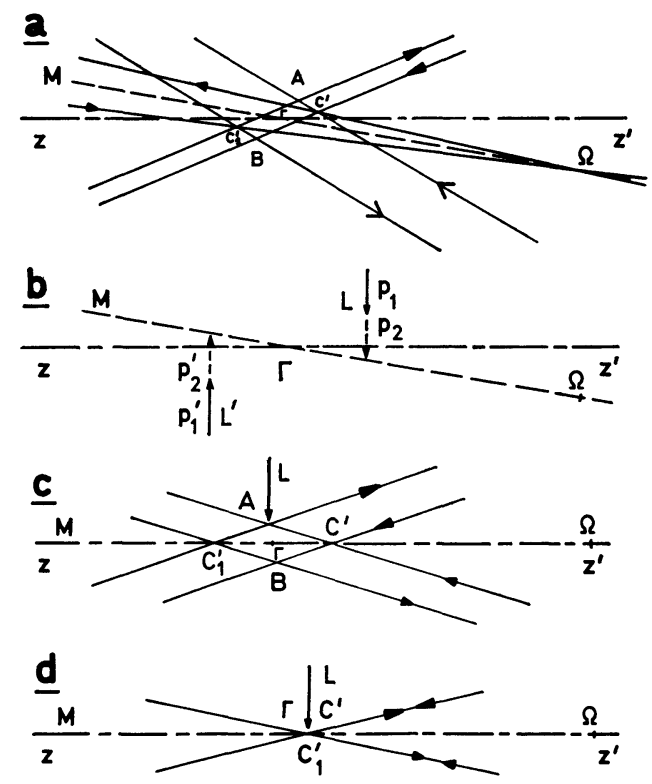

Fig. 6. - Recherche du centre $\Gamma$ du miroir électrostatique. $\mathbf{z}^{\prime} \mathbf{z}$ : axe principal du miroir ; $\mathrm{C}^{\prime}$ : centre du cross-over de sortie du «premier prisme»; $C_{1}^{\prime}$ : image de $C^{\prime}$ donnée par le miroir ; $\Omega$ : focale achromatique du "premier prisme", située sur son axe optique $\Omega M$.

Fig. $a$ : induction $\mathbf{B}$ non centrée, partie objet de l'analyseur non réglée.

Fig. $b$ : B est centrée avec la lame $\mathrm{L}:$ l'axe optique $\Omega M$ du prisme coïncide alors avec un axe secondaire du miroir convexe équivalent. $\mathrm{L}^{\prime}$ : image de $\mathrm{L}$ donnée par le miroir.

Fig. $c$ : induction $\mathbf{B}$ centrée, partie objet réglée pour amener $\Omega M$ sur l'axe $\mathrm{z}^{\prime} \mathrm{z}$.

Fig. $d$ : le déplacement en bloc du miroir amène $C_{1}^{\prime}$ sur $C^{\prime}$ et $\Gamma$. La position de $\mathrm{L}$ donne alors celle de $\Gamma$.

REVUE DE PHYSIQUE APPLIQUÉE. - T. 7, No 2, JUIN 1972 magnétique, c'est-à-dire par aligner l'axe optique de l'aimant avec un axe optique du miroir. Ensuite, on met à profit l'existence du champ de fuite pour amener l'axe optique de l'aimant sur l'axe principal du miroir (axe de révolution de la lentille qui joue le rôle de miroir) par réglage de la position de la partie objet de l'appareil : on se trouve alors dans le cas de la figure $6 c$. Il reste à déplacer le miroir pour amener $\mathrm{C}^{\prime}$ et $\mathrm{C}_{1}^{\prime}$ en coïncidence et trouver $\Gamma$.

\section{b) Méthode expérimentale.}

b.1. - Réglages préliminaires. - Ces réglages sont effectués en examinant l'extinction des taches directement sur le convertisseur représenté sur la figure 1, c'est-à-dire après la deuxième déflexion par le prisme magnétique.

- Centrage de l'induction magnétique. - On étudie pour cela l'extinction des taches sur l'écran fluorescent lorsqu'on déplace la lame $\mathrm{L}$ dans son propre plan, perpendiculairement à la direction de $\mathbf{B}$ (mouvement $\underline{2}$ Fig. 3) de manière à couper le faisceau et qu'on agit simultanément sur l'excitation de l'électro-aimant. Comme les trajectoires ioniques sont arrêtées à la fois par $\mathrm{L}$ et son image $\mathrm{L}^{\prime}$, pour un certain réglage, il ne subsiste plus sur l'écran que la tache centrale (ou plus exactement la ligne de taches perpendiculaire au plan radial), une variation très faible de $B$ dans un sens ou dans l'autre, fait alors disparaître cette tache de la même manière (Fig. 6b).

Réglage de la position de la partie objet (canon à ions + lentille à émission). - A la sortie de l'entrefer $\mathrm{du}$ "premier prisme ", on place une fente $\mathrm{F}$ de quelques millimètres de large dont les lèvres sont réglées (de l'extérieur de l'appareil) de manière à être parfaitement perpendiculaires au plan radial (plan de symétrie de tout l'appareil). Du fait du champ de fuite, l'axe optique réel du faisceau qui sort de l'aimant possède encore une légère courbure résiduelle et ne vient se confondre avec l'axe théorique $\mathrm{z}^{\prime} \mathrm{z}$ (qui serait l'axe du prisme si le champ de fuite n'existait pas) qu'à une certaine distance de l'aimant. Après réflexion, l'axe «image» suit le trajet symétrique de celui de l'axe « objet » par rapport à $\mathrm{z}^{\prime} \mathbf{z}$.

La fente $F$ (amovible) a une largeur telle qu'une fois le centrage réalisé, elle intercepte par le milieu les trajectoires 2 et 6 (l'une à l'aller, l'autre au retour). Le réglage consiste à déplacer légèrement dans le plan radial la partie objet de l'analyseur, à retoucher le centrage du champ magnétique de manière que les taches 2 et 6 apparaissent toutes deux coupées en leur milieu et de façon symétrique sur l'écran du convertisseur : les axes du prisme et du miroir sont alors en coïncidence (Fig. 6c).

b.2. - Recherche du centre du miroir. - Lorsque tout le système est ainsi parfaitement centré (cas de la figure $6 c$ ), on examine l'extinction des taches du plan radial symétriques par rapport à la tache entralec 
par l'un, puis l'autre bord du trou $\mathrm{T}$ de la lame $\mathrm{L}$ quand on déplace $\mathrm{L}$ suivant le mouvement 2 . Si le miroir est dans une position quelconque, $\mathrm{C}^{\prime}$ et son image $C_{1}^{\prime}$ sont distincts; en recherchant la position de L qui donne l'extinction simultanée par l'un, puis l'autre bord de $\mathrm{T}$ de deux taches symétriques par rapport à l'axe, on place la lame $\mathrm{L}$ dans le plan des points $\mathrm{A}$ et $\mathrm{B}$. Pour obtenir le centre $\Gamma$, on déplace alors le miroir, en retouchant la position de $\mathrm{L}$ jusqu'à obtenir l'extinction simultanée de la tache centrale et des deux taches symétriques. On est alors dans le cas de la figure $6 d$ où $\mathrm{C}^{\prime}, \mathrm{C}_{1}^{\prime}$, A et $\mathrm{B}$ sont désormais confondus avec le centre $\Gamma$ du miroir qui est matérialisé par la position de la lame $L$ : le centre du miroir est ainsi déterminé à quelques dixièmes de millimètre près $\left({ }^{3}\right)$.

On a cherché la position du centre $\Gamma$ par l'extinction des taches centrales, mais aussi pour les taches extrêmes correspondant aux rayons marginaux, ce qui a conduit à constater que les aberrations d'ouverture $\mathrm{du}$ miroir sont ici négligeables : en effet la distance du centre $\Gamma$ à la lentille-miroir reste la même quelles que soient les taches, centrales ou extrêmes dont on étudie l'extinction.

c) Déplacement du centre du miroir avec la tension de sa cathode. - Une fois la méthode de recherche $\mathrm{du}$ centre du miroir mise au point, nous avons pu étudier le déplacement de $\Gamma$ quand on fait varier la tension d'alimentation de la cathode du miroir. Cette migration du centre est loin d'être négligeable (quelques millimètres pour $\Delta V=10$ volts), et l'on observe sur l'écran un déplacement (une variation du grandissement) beaucoup plus sensible pour les taches du plan radial que pour les taches situées hors du plan radial. Mais, en fait, l'effet constaté ne vient pas tant du déplacement de l'image $C_{1}^{\prime}$ du cross-over que de la variation de pente des rayons.

En effet, si on suppose que $\mathrm{C}^{\prime}$ coïncide avec le centre $\Gamma \mathrm{du}$ miroir correspondant à une énergie $U_{0}$ des ions, pour des ions d'énergie $U=U_{0}+\Delta U$, le centre du miroir sera un point $\Gamma_{\mathrm{u}}$ voisin de $\Gamma$, mais distinct de $\mathrm{C}^{\prime}$. Après réflexion sur le miroir, les ions d'énergie $U$ seront focalisés en $\mathrm{C}_{\mathrm{u}}^{\prime}$, image de $\mathrm{C}^{\prime}$ et symétrique de $\mathrm{C}^{\prime}$ par rapport à $\Gamma_{\mathrm{u}} ; \mathrm{C}_{\mathrm{u}}^{\prime}$ sert de crossover d'entrée de la deuxième traversée du prisme pour les ions d'énergie $U$. A la sortie de l'analyseur, les ions d'énergie $U_{0}$ sont focalisés en $\mathrm{C}^{\prime \prime}$ et leurs trajectoires font un angle $\alpha_{i_{0}}$ avec l'axe tandis que les ions d'énergie $U$ sont focalisés en un point $\mathrm{C}_{\mathrm{u}}^{\prime \prime}$

(3) Il est à noter qu'une fois terminée la recherche du centre du miroir, le sommet n'est plus exactement sur la focale achromatique; il faut alors retoucher la tension du wehnelt pour placer convenablement le sommet. Ce dernier réglage décale légèrement la position du centre et une nouvelle retouche de celui-ci peut être nécessaire. Au bout de deux ou trois réglages successifs, on ne distingue plus de variation de la position des éléments optiques. dont la distance à $C^{\prime \prime}$ a été accentuée par la réflexion sur le miroir, et leurs trajectoires font avec l'axe un angle $\alpha_{i_{u}}$ différent de $\alpha_{i_{0}}$. Le grandissement angulaire est donc très sensible aux différences d'énergie des ions.

Ceci correspond aux problèmes que nous avions déjà évoqués [3] $\left({ }^{4}\right)$ pour l'utilisation du miroir avec des ions secondaires présentant une certaine dispersion d'énergie: il y aura en conséquence une certaine «dispersion du centre » du miroir le long de l'axe, et sur l'écran, une superposition d'images ioniques de grandissements différents, ce qui pourrait conduire à une perte de résolution ou tout au moins à amoindrir la qualité des images.

V. Conclusion. - Nous avons mis au point une méthode pratique pour déterminer un certain nombre de caractéristiques optiques de l'analyseur ionique : position des points stigmatiques et du centre du miroir électrostatique, réglage de la position de l'axe de la partie objet et de l'achromatisme du système global. De plus, le dispositif que nous avons utilisé est assez précis pour mettre en évidence les aberrations d'ouverture du prisme magnétique et l'influence de l'énergie des particules sur la position du centre du miroir.

Cette étude expérimentale a permis de mieux situer les problèmes liés à la recherche d'une amélioration du pouvoir séparateur sur les images de distribution. Ainsi que nous l'avions déjà indiqué [3], la variation de la position du sommet du miroir avec l'énergie des particules pouvait être responsable d'un astigmatisme au niveau de l'image filtrée. Mais il est également clair que la variation de la position du centre avec l'énergie des particules se traduira dans la deuxième traversée du prisme par une variation de la position du cross-over de sortie qui peut entraîner, au niveau de l'image filtrée, des grandissements variant avec l'énergie des particules et, de ce fait, un « chromatisme de champ ». Quoiqu'il en soit, la difficulté consiste à découpler chaque effet et à estimer son ordre de grandeur.

Avec l'appareil dont nous disposons, nous ne pourrons poursuivre les études sur l'amélioration de la qualité des images de distribution qu'après nous être assurés que le champ magnétique est suffisamment homogène. Néanmoins, le positionnement correct de la fente de sélection a rendu possible le réglage de sa largeur à une valeur optimum. On peut ainsi réduire la largeur de la fente de façon à obtenir la meilleure résolution en masse possible compte tenu des diaphragmes d'entrée et d'ouverture sans pour cela affecter l'uniformité d'éclairement de l'image.

(4) Dans la référence [3], nous supposions le centre du miroir fixe, et la position de son sommet variait avec l'énergie des ions, alors que dans le cas présent, c'est le sommet du miroir qui reste fixé. 


\section{Bibliographie}

[1] Leleyter (M.), Slodzian (G.), Rev. Phys. Appl., [4] Matsuda (H.), Progress Report of the International 1971, 6, 65.

[2] Castaing (R.), Hennequin (J.-F.), Henry (L.) et SLODZIAN (G.), dans Focusing of charged particles, 2, éd. Septier (A.), 1967, Academic Press, New York.

[3] Leleyter (M.), Slodzian (G.), VII e Congrès international de microscopie électronique, Grenoble, 1970, 35, éd. Favart (P.). Conference on Mass Spectrometry, Bruxelles, 31 août-4 septembre 1970 .

[5] ENGE (H.-A.) dans Focusing of charged particles, 2, éd. Septier (A.), 1967, Academic Press, New York.

[6] WollniK (H.), Nucl. Instr. Meth., 1967, 53, 197.

[7] Slodzian (G.), Thèse de Doctorat, 1963, Paris ; Ann. de Phys., 1964, 9, 591. 